le portiQue Le Portique

Revue de philosophie et de sciences humaines

17 | 2006

Marcel Mauss et les techniques du corps

\title{
Hétéro-réflexivité des techniques du corps.
}

L'épistémologie physio-psycho-sociale de Marcel Mauss

\section{Bernard Andrieu}

\section{CpenEdition}

Journals

Édition électronique

URL : http://journals.openedition.org/leportique/779

DOI : $10.4000 /$ leportique. 779

ISSN : $1777-5280$

Éditeur

Association "Les Amis du Portique"

Édition imprimée

Date de publication : 1 janvier 2006

ISSN : 1283-8594

Référence électronique

Bernard Andrieu, « Hétéro-réflexivité des techniques du corps. », Le Portique [En ligne], 17 | 2006, mis en ligne le 15 décembre 2008, consulté le 25 mars 2021. URL : http://journals.openedition.org/

leportique/779 ; DOI : https://doi.org/10.4000/leportique.779

Ce document a été généré automatiquement le 25 mars 2021.

Tous droits réservés 


\title{
Hétéro-réflexivité des techniques du corps.
}

\author{
L'épistémologie physio-psycho-sociale de Marcel Mauss
}

\section{Bernard Andrieu}

" Je crois même que toutes les découvertes récentes en réflexothérapie méritent notre

attention..." "

Marcel Mauss, 1936.

1 Soixante-dix ans après, le texte sur les techniques du corps, si souvent comparé avec les «Techniques de soi » du dernier Michel Foucault, pourrait être aussi lu du point de vue de la réflexivité corporelle du sujet social plutôt qu'individuel, comme un modèle épistémologique sur les conditions de réflexothérapie à l'heure où chacun se précipite dans les bras du développement personnel ${ }^{1}$. Le corps à corps inhérent au processus de socialisation est une expérience double d'incorporation et d'ajustement sans laquelle le sujet corporel serait condamné à trouver en lui ce que l'éducation corporelle lui a apporté de l'extérieur. Les techniques du corps sont un moyen de sortir de l'opposition stérile entre internalisme et externalisme en posant l'interaction bioculturelle, et pas seulement symbolique, comme un principe de constitution et de réflexivité corporelle.

Comme l'habitus est de nature sociale, Mauss ajoute à Kant une critique de la raison pratique collective $^{2}$. Si nous parvenons à distinguer un Anglais d'un Français à la position des mains qu'ils adoptent à table, c'est en raison de l'ensemble des éléments psychologique, biologique et sociaux du «fait total» des techniques du corps. Daniel Dennett a démontré combien l'hétéro-phénoménologie ${ }^{3}$ était nécessaire pour supposer au sujet une posture intentionnelle dont il n'a pas immédiatement conscience. Yves Clos ${ }^{4}$, avec «l'instruction au sosie ", a démontré comment l'auto-réflexivité d'un expert sur son propre savoir est rendu impossible par une sorte de point aveugle qui nécessite une série de médiations. Pierre Parlebas pose alors l'hypothèse selon laquelle les schémas corporels peuvent être pluriels en raison des situations/contextes d'action. Les techniques du corps seraient ainsi dépendantes de «tactiques» corporelles ${ }^{5}$ se produisant lors de l'action motrice. 
Dès lors comment Mauss a-t-il apprit à nager sans s'en rendre compte par lui-même ? Pourquoi a-t-il fallu qu'il lise un article, qu'il regarde des films au cinéma, qu'il remarque des démarches et des gestes pour qu'il s'aperçoive comme constitué par des habitudes corporelles d'un autre temps? Ou alors est-ce seulement l'observation des jeunes corps qui lui fait découvrir la vieillesse de ses apprentissages?

L'irréductibilité

Dans le cadre de l'ethnologie descriptive qu'il veut défendre, M. Mauss rencontre les techniques du corps comme un argument essentiel : en effet « il y a toujours un moment où la science de certains faits n'étant pas encore réduite en concepts, ces faits n'étant pas même groupés organiquement, on plante sur ces masses de faits le jalon d'ignorance: "Divers", c'est là qu'il faut pénétrer $»^{0}$.

5 La raison de cette irréduction, sinon irréductibilité, des techniques du corps à des gestes conscients provient d'abord de ce qu'il y a des techniques du corps plutôt qu'une théorie de la technique du corps. En effet, la théorie de la technique du corps serait une conceptualisation abstraite qui ne pourrait rendre compte des façons dont les hommes, dans l'action, "savent se servir de leur corps». Une ethnologie théorique du corps n'a pas encore le sens que lui donnera le structuralisme, car l'étude "société par société", conduit moins à la dispersion factuelle qu'une théorie pourrait unifier en système symbolique, qu'à une "exposition » des "façons » variées de se servir du corps.

6 Puisque, selon une méthode inductive empiriste, « il faut procéder du concret à l'abstrait et non pas inversement", en accordant aux variétés du terrain ethnologique une fécondité épistémologique, celle de "linconnu» qui "se trouve aux frontières des sciences", reconnaître «qu'on ne sait pas » est le jalon d'ignorance nécessaire à la curiosité moins exotique que méthodologique de l'ethnologie des techniques du corps. «Une description pure et simple des techniques du corps » n'a pas besoin d'être précédée d'une théorie dans laquelle il faudrait les inclure en les classant; mais l'abstraction du concept des techniques du corps conduit Mauss moins à une typologie qu'à une réflexion théorique sur les procédés (habitudes, réflexivités, apprentissages, éducations, incorporations). Le terrain n'est donc pas réductible, il sert de matériaux à une théorisation des processus.

7 La distinction entre technique et enseignement technique est déjà engagée par Mauss en 1927 dans son texte sur Divisions et proportions des divisions de la sociologie: car la division des phénomènes de la physiologie sociale sépare deux groupes de faits: les représentations collectives et les pratiques collectives. La science et la technique forment une "unité naturelle ${ }^{0}$, le lien entre enseignement technique et éducation corporelle concrétise l'incorporation. En 1929, dans un débat lors d'une séance de L'institut français d'Anthropologie à propos du film de P. Guillaume et I. Meyerson sur la psychologie des singes, M. Mauss distingue bien le bâton « moyen » et le bâton " outil », mettant l'accent sur l'étude de "la capacité de se servir des choses... le tout naturellement sans dressage. Cela instruirait sur les origines de la technologie humaine ${ }^{0}$. Cette différence entre capacité et façon de se servir, si elle n'existe pas avec les techniques du corps observés dans les sociétés humaines, pose la question.

L'éducation corporelle

8 En cherchant à qualifier les éducations spéciales, qu'il sépare de l'éducation générale, Marcel Mauss n'étudie pas l'initiation et l'éducation des filles mais celles des garçons. Leur éducation consiste en une série d'épreuves là où l'initiation, qui sépare les sexes, les soumet à une "éducation intensive " ${ }^{\circ}$. Six types d'éducations spéciales sont distinguées: 1. L'enseignement des techniques du corps; 2. L'enseignement des 
techniques manuelles ; 3. Les traditions techno-scientifiques ; 4. Éducation esthétique ; 5. Éducation économique ; 6 . Juridique et religieuse.

9 Si ces éducations participent de la transmission de la cohésion sociale, seules les techniques du corps sont un perfectionnement là où les cinq autres types d'éducations spéciales sont plutôt des instructions et des apprentissages de connaissance.

10 L'autorité traditionnelle pour le corps ne repose plus seulement sur l'a priori social mais sur l'a posteriori social. L'éducation se superpose à l'imitation, devenant une sorte "d'imitation prestigieuse ${ }^{0}$, car elle repose sur le modèle et la métaphore mécanique de "l'enchaînement " afin de dépasser le dogme comtien de la séparation entre le social et le biologique. Si les mouvements du corps reposent sur l'appareil biologique et physiologique, l'enjeu pour Mauss est d'expliquer "quelle est l'épaisseur de la roue d'engrenage psychologique? Je dis exprès roue d'engrenage ${ }^{0}$. Les faits psychologiques ne sont pas des causes, au sens positiviste du terme, mais des engrenages. Mauss cherche à déplacer les frontières non seulement de la physiologie mais de la psychologie tant dans une référence au stoïcisme et ces techniques ascétiques de maîtrise du corps par l'esprit («l'éducation du sang froid ${ }^{0}$ du stoïcisme comme de l'alpinisme) que par une description "de phénomènes biologico-sociologiques ». Il défend la thèse qu'il s'agit de "montages physio-psycho-sociologiques de séries d'actes ${ }^{0}$ définissant ainsi un apprentissage par modification de l'appareil biologiques et physiologique par l'action de la culture.

11 La bio-sociologie, à l'inverse de la sociologie biologique raciale et eugéniste, met l'accent sur "l'éducation des races qui se sélectionnent en vue d'un rendement déterminé " ${ }^{0}$. La distinction et la progression de l'éducation du sang-froid séparerait, dans une sorte de civilisation des mours, les mécanismes "de retardement, d'inhibition de mouvement désordonnés ${ }^{0}$ qui développent une résistance à l'émoi envahissant comme preuve d'un contrôle des réactions tant dans la vie sociale que mentale. L'éducation du sang-froid caractérise les différentes initiations stoïciennes : résistance, sérieux, présence d'esprit, dignité, conscience claire.

L'éducation du sang-froid, incorporée par Mauss dans ce qu'il appelle son «alpinisme d'autrefois", lui évite le vertige par la maîtrise du corps lui permettant «de dormir debout sur le moindre replat au bord de l'abîme ${ }^{0}$. Ainsi la maitrise du corps n'est pas semblable à l'auto-réfléxivité de sa technique du corps, elle est seulement une conscience de l'habitus mais n'est pas suffisante pour s'en débarrasser. La maîtrise du corps est un savoir opératoire du soi plutôt qu'une technique de réflexivité.

L'auto-réflexivité corporelle

13 Mauss constate des «progrès fabuleux ${ }^{0}$ de l'alpinisme pendant son existence, accédant ainsi à une conscience historique de la modification des techniques, alors même qu'il ne parvient pas à changer sa propre technique, comme si un point aveugle sembler s'attacher à l'incorporation d'habitus. Cet accès à un corps enfoui se fonde sur la nécessité de retrouver "des sensations, des émotions " ${ }^{\circ}$. La difficulté est de s'apercevoir de ce qui constitue la prise habituelle du monde et des autres, à travers le corps. La difficulté est l'absence de toute phénoménologie de la perception, et notamment, de la dualité du schéma-corporel et des images du corps pourtant contemporain (en 1923 et en 1935 chez Schilder, et dans la conférence de 1934 de Maurice Merleau-Ponty sur le primat de la perception).

14 Qu'une réflexivité du corps soit nécessaire pour parler des techniques du corps ne conduit pas forcément, dans la métacognition, et autre principe transcendantal, au 
dédoublement méthodique! Nick Crossley $^{0}$ démontre comment les techniques réflexives du corps reposent sur la réflexivité sociale du corps. Celle-ci est à ce point incorporée qu'elle a transformé et modelé le corps dans sa matière, dans ses formes et dans ses modes d'actions. Il faut distinguer l'autoréflexivité sociale de l'auto-réflexivité individuelle comme si la première devait être la condition de possibilité de l'exercice de la seconde.

L'idée d'un apprentissage inconscient est établie sans que l'incorporation puisse être décrite comme telle par les penseurs de la fatigue, de l'effort, de l'exercice et de l'habitude. Ainsi Marie Léon Coste dans un livre, justement remarqué par Pierre Janet dans la Revue Philosophique en 1889, rappelle combien au sujet de l'inconscient «toute impression extérieure est l'occasion d'une modification interne du système nerveux " ${ }^{0}$, car "l'éducation a développé dans l'organisme une manière d'être spéciale à l'un ou l'autre type... chez le marcheur, l'être inconscient rend la marche naturelle... il faut que l'être inconscient subisse les nouvelles impressions, se modifie, pour avoir le travail musculaire facile » ${ }^{0}$.

Cette irréversibilité de la technique non naturelle du corps serait à l'origine de la dialectique, selon Muriel Darmon et Christine Detrez ${ }^{0}$, entre "corps dur » et " corps mou ». Pourtant Mauss ne s'en aperçoit pas lui-même, comme si la conscience de sa propre technique corporelle, de nage, dépendaient d'un "point de départ, [d']un cadre d'observation » fourni par un autre que soi-même. En l'occurrence, la lecture de l'article « Nage» dans l'édition de 1902 de la British Encyclopaedia devait conduire " dans la suite ", c'est-à-dire dans les années 1930, à un cadre d'observation des techniques du corps. Ce cadre d'observation porte sur la distinction opératoire dans les actes corporels de la technique (de la plongée) et de la technique de l'éducation (de la plongée).

L'irréversibilité

17 La différence entre Schilder et Mauss serait due, selon Pierre Arnaud et Gérard Broyer, à un "niveau épistémologique différents des auteurs" ${ }^{\circ}$. Si Schilder, comme Lhermitte, Wallon et Lacan, cherche à échapper à un réductionnisme des schémas mécanistes du corps en préservant sa plasticité. Mauss observe et repère, seulement, les différentes manières dont les hommes utilisent leur corps. Cette opposition entre structure du développement et ethnohistoire des modes corporels relève de deux paradigmes de l'enculturation motrice. D'une part, dans la biologie du développement, l'image du corps dépend de la physiologie du schéma corporel et du degré de l'activité perceptive du bébé dans son interaction constitutive avec son environnement. D'autre part, les techniques du corps sont acquises par imitation éducative qui opère une mise en forme $\mathrm{du}$ corps. Pour Mauss, l'acte à imiter «s'impose du dehors, d'en haut, fût-il un acte exclusivement biologique concernant son corps ", là où l'interaction écologique met plutôt l'accent sur l'activité perceptive dans la constitution du soi.

Les techniques du corps sont un perfectionnement définitif de "l'éducation physique commencée ${ }^{0}$. Cette notion de perfectionnement définitif indique à la fois un achèvement et une incorporation en habitus dont la décorporation révèle le caractère irréversible. Si Mauss reconnaît une plasticité initiale, il découvre, face à l'évolution de la nage de la brasse au crawl, qu'on " a perdu l'usage d'avaler de l'eau et de la cracher ». Mais cette perte de l'usage repose sur de nouvelles techniques que le sujet ne peut pas perdre sans se décorporer, sans sortir de son «temps » : "les nageurs se considéraient, de mon temps, comme des espèces de bateaux à vapeur. C'était stupide, ainsi enfin, je fais encore ce geste, je ne peux pas me débarrasser de ma technique ». 
19 L'impossible décorporation individuelle est contradictoire avec la plasticité de l'apprentissage et l'efficacité de la répétition dans les exercices corporels dont Mauss, comme caporal, a pu mesurer l'efficacité de la conscience de groupe. Les séries d'actes physio-psycho-sociologiques prouvent l'efficacité de l'autorité sociale :

« caporal $^{0}$, voici comment j'enseignais la raisons de l'exercice en rang serré, la marche par quatre et au pas. Je défendais de marcher au pas et de se mettre en rang et en deux files par quatre, et j'obligeais l'escouade à passer entre deux des arbres de la cour. Ils se marchaient les uns sur les autres. Ils se sont rendu compte que ce qu'on leur faisait faire n'était pas si bête. Il y a dans tout l'ensemble de la vie en groupe une espèce d'éducation des mouvements en rang serré ${ }^{0}$.

Si l'autoréflexivité individuelle rend impossible la réversibilité de l'habitus, préparant ainsi la thèse de la reproduction sociale, la pratique corporelle collective favorise l'hétéroréflexivité : "une espèce d'éducation des mouvements " par une incorporation plutôt qu'une imitation. L'ajustement produit une mise en cohérence des gestes, des pas, comme dans la marche en rang serré. Ainsi, «les circonstances de la vie en commun, $d u$ contact $"{ }^{0}$ démontrent qu'il y a une adaptation susceptible d'être incorporé qui ne relève plus seulement d'une psychologie individuelle.

21 L'irréversibilité individuelle est une thèse peu compatible avec celle de l'habitus que met en avant, contre Georges Dumas, Mauss pour démontrer la supériorité de l'éducation par rapport à l'imitation, car il faut y voir « des techniques et l'ouvrage de la raison pratique collective et individuelle ${ }^{0}$.

L'exemple de la natation

22 Le contexte d'écriture et d'invention des techniques du corps doit être situé pour comprendre l'originalité de Mauss. Georges Vigarello estimait que cette notion avait vieilli par " la mise en évidence de cohérences entre certaines catégories de gestes et surtout, celle de complicités entre des "gestualités" et des "mentalités" " 0 notamment en natation. Mauss précise :

« En 1898, j'ai été lié à quelqu'un dont je connais bien encore les initiales, mais dont je ne me souviens plus du nom. J'ai eu la paresse de le rechercher. C'était lui qui rédigeait un excellent article "Nage" dans l'édition de la British Encyclopedia de 1902 alors en cours... Il m'a montré l'intérêt historique et ethnographique de la question. Ce fut un point de départ, un cadre d'observation ${ }^{0}$.

23 Dans son Paris dans l'eau, écrit en 1844, l'écrivain Eugène Briffault (1799-1854) ne recommandait que la brasse dans la Seine et surtout de «ne pas oublier de tenir la tête élevée " ${ }^{0}$. En 1905, Paul Augé rédige l'article " Natation " pour le Larousse ${ }^{0}$. La période autour des jeux olympique de 1900 à Paris est propice à une évolution des techniques. Mauss écrit dans un contexte de fondation de la nage moderne et de modification des techniques confrontant la nouvelle génération des nageurs anglais et américains.

24 Pour l'English Side Stroke, cette «brasse sur le côté», se complexifie vers 1840 en accentuant la perte de symétrie dans les mouvements de bras. L'un travaille davantage en poussant et l'autre en traction (retour sous-marin). Le corps est incliné (tête hors de l'eau) et les jambes passent d'une gestuelle de grenouille à un ciseau qui coince l'eau entre elles. La première piscine intérieure a été construite en 1862 en Angleterre. C'est en 1873 que John Arthur Trudgen (1860-1940), un nageur anglais, réintroduit the front crawl en Angleterre sur la base d'observations qu'il a faites en 1870 dans un voyage en Amérique du Sud auprès des Native Americans. C'est une combinaison de la brasse avec le retour alternatif des deux bras (style coup de poing). Le nageur prend appui sur tout l'avant-bras pour tirer le corps à lui. Les mouvements de bras ne sont accompagnés que 
d'un faible mouvement des jambes ressemblant à une ondulation du corps à l'oblique. Le corps est moins incliné que pour les nages précédentes, mais les contraintes respiratoires qu'il crée, font qu'il n'est utilisable que sur de courtes distances. En 1898, William Kennedy-Laurie Dickson (1860-1935) réalise le premier film sur la natation en water polo (68 minutes). Il est réalisé durant un voyage à Worthing entre Brighton et Hove. Au J.O. de 1900, les nageurs s'affrontent à Asnières, dans la Seine. Au programme, six épreuves individuelles et un relais. Sur l'une d'elles (60m sous l'eau) la France obtient sa première médaille d'or. Coté style, la mode n'est plus au Sidestroke, mais au trudgen (crawl). F. V. C. Lane est l'un des premiers a utilisé cette nage. Il gagne ici deux courses, et en 1901, il porte le record du monde du 100 Yard à une minute.

À la fin du xix ${ }^{e}$ siècle se mettent en place des fédérations. En France, la commission natation de l'USFSA (Union des sociétés françaises de sports athlétiques créés en 1889) reprend les règles établies par l'A.S.A. (Amator swimming association) anglaise en 1880. La FINA (Fédération internationale de natation amateur) est créée en 1908. Harry Hebner (1891-1968) est le premier à passer du dos brassé au dos crawlé (mouvement alternatif des jambes) en 1912. Le terme crawl (ramper) vient de nageurs australiens qui, pour améliorer le double over arm stroke, ont remplacé le mouvement de jambe par un battement alternatif partant du genou. Puis, Duke Kahanamoku (1890-1968), un américain, transforma le même battement en le faisant partir de la hanche (années 1910). Johnny Weissmuller (1904-1984), en 1922, « cassa » la barre de la minute au 100m nage libre. Il révolutionna cette nage et la natation en général en commençant à rythmer la respiration sur les mouvements propulsifs des bras. Transformations qui impliquent une nage beaucoup plus hydrodynamique et limite donc les résistances à l'avancement. En 1926, Erich Rademacher (1906-1970), un allemand, utilise le papillon brassé lors des derniers mouvements d'une épreuve de brasse (retours aériens des deux bras). En 1933, l'Américain Henry Meyers nage dans un style que l'on nomme «butterfly».

En ce qui concerne les techniques de plongée et de leur éducation, en 1926, un officier de marine essaie un bloc autonome dans une piscine. Ce bloc est constitué d'une bouteille de gaz et d'un régulateur. L'air y arrive en continu. Cela est possible grâce à un système de vis-pointeau. Cet officier de marine, Yves Le Prieur (1885-1963), est considéré comme le père de la plongée, il est aussi à l'origine de nouveaux matériels, comme un vêtement de plongée à réserve d'eau chaude (tout confort, surtout pour le nord de la France...), de caissons étanches dans lequel on peut transporter un appareil photo, d'une cage anti-requins... En 1933, les palmes apparaissent, et rendent le plongeur plus mobile. Dans ce contexte d'acceptation de l'eau indépendamment de la nage, il s'agit de familiariser, de dompter, d'inhiber, de créer une assurance, d'exercer son corps et notamment les yeux par une sélection motrice, contre l'instinct des arrêts, des mouvements et des gestes. Comme Jean Le Camus ${ }^{0}$ l'a démontré, c'est durant les années 1960 que débutent les pratiques aquatiques du bébé, sous l'impulsion des pratiques périnatales et postnatales de Frédéric Leboyer, Michel Odent, I. Tcharkovksi et $D$. Bousse.

Nager, Marcher, méthode naturelle ou technique rationnelle?

Thierry Terret en 1991 a démontré dans sa thèse comment en France ${ }^{0}$, de 1877 à 1914, une natation gymnique est une natation à la fois en tant que gymnastique préparatoire et un exercice d'application d'obédience militaire (maintien de l'ordre). Jusqu'à la première guerre mondiale, la natation relève d'un apprentissage de gestes et de 
techniques de bases (brasse, nage sur le dos, plongeon simple), objets de formation au service de finalités disciplinaires (former des individus sains, virils, soumis, dont le pays a besoin économiquement et militairement et utilitaires ${ }^{\circ}$ ).

De 1919 à 1959 se développe une natation pratique en lien avec le règlement général de la méthode française (1925-1932). Avant d'être une technique du corps, la natation ou la marche est l'objet, comme toute éducation physique, d'un débat qui oppose les partisans de la méthode naturelle et ceux de la technique rationnelle. Soit le corps a des mouvements naturels qu'il convient de retrouver comme les peuples primitifs l'avaient initialement découverts (on voit ici que l'ethnologie descriptive n'a pas atteint Hébert), soit le corps dépend de notre appareil locomoteur, de la maîtrise de soi-même et de l'image mentale du mouvement selon la physiologie de G. Demeny. Si le geste était produit uniquement par la physiologie, la technique serait déjà contenue dans le corps au point qu'il ne faudrait que suivre son instinct ${ }^{\circ}$.

Paul Beulque (1877-1943) va se baigner dans les fossés de la Cense Montagne où il devient le champion de la patte de chien, technique de nage qui devint plus tard le crawl. Il se rend aussi le dimanche à la piscine des bains roubaisiens et à l'école de natation du Pont Morel. Il fonde en 1904 les Enfants de Neptune de Tourcoing et est nommé moniteur chef de la piscine dès son ouverture. Il invente entre 1919 et 1923, une méthode où la brasse est la nage de base. Son apprentissage est fondé sur une décomposition du geste à quatre temps, en dehors de l'eau puis dans l'eau accroché à une potence, en référence à G. Demeny, et en opposition à Georges Hebert et à sa méthode naturelle. Dans cette période d'après guerre, la vocation disciplinaire de la natation s'estompe au bénéfice d'une cause plus utilitariste: apprendre à ne pas se noyer, donner le minimum pour survivre...

Georges Hébert (1875-1957) º de 1895 à 1903, « parcourt le monde sur les derniers voiliers de la marine française, ce qui lui permet de découvrir d'autres peuples, d'autres races, d'autre modes de vie... Là aussi nous avancerons une hypothèse: les voyages à travers le monde étant souvent la cause de révolutions intérieures, il n'est pas impossible que notre jeune officier - il avait vingt ans seulement au départ de ce long voyage, se soit remis en cause au point d'orienter sa vie dès son retour en France $"{ }^{0}$. Il aura pu y constater que ces peuplades doivent leur forme à l'exercice physique quotidien qu'elles pratiquent en accomplissant les gestes naturels nécessaires à leur subsistance: courir, sauter, lancer, porter, nager, etc. Préoccupé d'éducation, G. Hébert met alors au point et codifie une méthode d'éducation physique basée sur des gestes naturels qu'il fait connaître sous le nom de "Gymnastique selon la Méthode Naturelle ». Dès 1904, il est autorisé à appliquer ses principes à un groupe de 1200 hommes et, en 1905, il introduit l'usage de la «fiche de performances cotées » basée sur cinq épreuves, fiche qui permet de constater l'évolution des résultats de l'exercice. Il perfectionne et révolutionne l'entraînement physique et surtout lui confère un but altruiste convenant bien aux militaires en temps de paix: sauvetage en mer, transport de blessés, secours lors de catastrophes.

31 En 1909, Hébert est chargé de faire appliquer la méthode naturelle à tous les services de la Marine. Durant la guerre 1914-1918, il jouera un rôle prépondérant dans la mise en condition physique de l'armée où sa méthode est généralisée. Hébert développe les principes de la Méthode Naturelle dans plusieurs livres dont son maître-ouvrage publié en 1936, "L'éducation Physique, virile et morale par la Méthode Naturelle». Bien que comptant des détracteurs, cette méthode fait son chemin et des Centres hébertistes se créent un peu partout en France. Arrive la guerre de 1940. Le gouvernement du 
Maréchal Pétain adopte la Méthode Naturelle pour l'éducation physique des jeunes. Pour la natation ${ }^{0}$, il va plus loin et différencie des nages essentielles (brasse et dos brassé) et des nages d'évolutions (se laisser flotter) où l'équilibre du corps est proche de la verticale, des nages de propulsion, ou nages sportives (crawl, dos crawlé, brasse coulée) où l'équilibre du corps est alors horizontal.

La Méthode physiologique de Georges Demeny (1850-1917) commence elle en 1880, lorsque, moniteur de la société de gymnastique "La Nationale », il fonde le "Cercle de gymnastique rationnelle» où vont se développer les travaux de ceux qui veulent, dans une perspective explicitement positiviste, former des "ingénieurs biologiques", capables de perfectionner la "race humaine ». Lancée en 1873, l'Union des sociétés de gymnastique de France (USGF) soutient la politique républicaine en organisant de nombreux concours de (ou à) caractère martial et patriotique. Ces manifestations ont pour but, après la défaite de 1870 , de restaurer chez la jeunesse le culte de la patrie et de renforcer l'unité nationale. L'USGF ne cesse de prospérer et compte 260 sociétés en 1883, 809 en 1909. Georges Demeny, assistant d'Étienne-Jules Marey, invente le phonoscope, ancêtre de la caméra. Scientifique et sportif, ce précurseur du cinéma s'intéressa particulièrement à son sujet favori, le sport, dans les nombreux films qu'il réalisa. Son travail s'est inspiré de l'étude de la décomposition du mouvement et du geste. L'expérimentation de la marche et de la course affirme le point de vue physiologiste et met l'accent sur l'efficacité de l'organisme, à partir d'une réflexion sur l'économie dans le déplacement. Dans ses recherches sur É. Marey et G. Demeny, Christian Pociello note que si la gymnastique s'était proposée d'atteindre des objectifs hygiéniques et esthétiques, il revint au $\mathrm{XIX}^{\mathrm{e}}$ siècle, industriel et technique, d'introduire les aspects économiques du corps humain en termes de rendement et d'ergonomie: comment le corps résout-il le problème de l'économie dans le déplacement?

Là où Marcel Mauss affirme que la marche est un «habitus du corps " ${ }^{0}$, le laboratoire d'Étienne Marey et Georges Demeny établit une science du mouvement. L'esthétique du mouvement repose sur un principe d'économie naturel qui produit santé, beauté, adresse et virilité, le réalisme photographique rendant compte du découpage des gestes, des postures et des modes d'action du corps en mouvement. Pourtant dans le Manuel d'ethnographie en 1947, Mauss précise que «les techniques du corps seront étudiées à l'aide de la photographie et si possible du cinéma au ralenti ${ }^{0}$. Les techniques ${ }^{0} \mathrm{du}$ chronophotogramme géométrique du marcheur, de l'analyse expérimentale, cinématique et dynamique des allures normales de l'homme (marche, course, saut), des images stéréoscopiques de la trajectoire, étaient réalisés entre 1883 et 1896 par Marey et Demeny.

Conclusion

Les techniques du corps sont un concept inter-scientifique qui offre une alternative à la dispersion épistémologique $d u$ corps dans des disciplines séparées. Montage et enchaînement psycho-bio-social, les techniques du corps sont un pont jeté entre psychologie et sociologie, entre individu et collectif à un moment où la domination de la psychologie individuelle pouvait faire croire à la stérilité de la sociologie.

Mauss contribue pourtant à ouvrir l'épistémologie du corps par l'interaction bioculturelle en situant l'incorporation du social dans la modification de la matière du sujet. 


\section{NOTES}

1.. B. ANDRIEU, Toucher. Épistémologie du soin du soi, Paris, Belles lettres, coll. « Médecine et sciences humaines ", 2006.

2.. M. MAUSS, « Les techniques du corps », Sociologie et anthropologie, Paris, PUF, p. 369.

3.. D. DENNETT, La Méthode de l'hétérophénoménologie. La conscience expliquée, trad. Pascal Engel, Paris, Odile Jacob, 1993 [1991], p. 103.

4.. Y. СLOT, La Fonction psychologique du travail, Paris, PUF, 1999. Y. CLOT, D. FAITA, « Genres et styles en analyse du travail. Concepts et méthodes ", Travailler, 4, 2000.

5.. P. PARLEBAS, « Les tactiques du corps », in M.-P. JULIEN et J.-P. WARNIER (sous la dir. de), Approches de la culture matérielle. Corps à corps avec l'objet, Paris, l'Harmattan, 1999,

p. 29-43.

0.. Op. cit., p. 365.

0.. M. MAUSS, «Divisions et proportions des divisions de la sociologie », CEuvres, tome 3, Paris, Minuit, 1969, p. 179 [1927].

0.. M. MAuss, « Débat sur l'origine de la technologie humaine », dans đEuvres, tome 3, Éd. Minuit, p. 257 [1929].

0.. M. MAUSS, « Fragment d'un plan de sociologie générale descriptive. Classification et méthode d'observation des phénomènes généraux de la vie sociale dans les sociétés archaïques (phénomènes généraux spécifiques de la vie intérieure de la société) », dans CEuvres, tome 3, Paris, Minuit, 1969, p. 343 [1934].

0.. TC, p. 369.

0.. TC, p. 384.

0.. TC, p. 385.

0.. TC, p. 384.

0.. TC, p. 385.

0.. Ibid.

0.. Ibid.

o.. TC, p. 381.

0.. E. PERRIN, « Représentations et images du corps : quelques réflexions sur les nouvelles pratiques du corps ", Anthropologie des techniques du corps, Revue STAPS, 30 octobre, p. 63-72, ici p. 66, 1984.

0.. N. CRossley, "The Circuit Trainer's Habitus : Reflexive Body techniques and the Sociality of the Workout", Body \& Society, vol. 10 (1), 2004, p. 37-69. En particulier, p. $38-40$.

0.. M. L. coste, L'Inconscient. Étude sur l'hypnotisme, Paris, Baillière, 1889, p. 23.

0.. Op. cit., p. 59-60.

0.. M. DARMON et C. DETREZ partent de l'évidence du corps naturel : « Avant-propos », Corps et société. Problèmes politiques et sociaux, 2004, p. 9.

0.. P. ARNAUD, G. BROYER, « Des techniques du corps aux techniques sportives », dans p. ARNAUD, G. BROYER Eds, La Psychopédagogie des activités physiques et sportives, Éd. Privat, p. 135-157, ici p. 13 [1983].

0.. TC, p. 344.

0.. TC, p. 367. 
0.. Lors de la Grande guerre, que Mauss, engagé volontaire, effectue comme interprète. Beaucoup disparurent dans les tranchées, dont le fils de Durkheim.

0.. TC, p. 384.

0.. TC, p. 385.

0.. TC, p. 369.

0.. G. VIGARELLO, «Introduction ", Anthropologie des techniques du corps, Revue STAPS, 30 octobre 1984, p. 17-25, ici p. 21. G. Vigarello parlera plutôt de technique corporelle dans son article : G. VIGARELLO, J. VIVES, « Technique corporelle et discours technique », Revue EPS, $1983, \mathrm{n}^{\circ} 184$.

0.. TC, p. 366.

0.. Merci à J.-F. Bert pour m'avoir trouvé cette référence : E. BRIFFAULT, Paris dans l'eau, Paris, Éd. J. Hetzel, 1844, p. 45.

0.. P. AUGÉ, « La natation », Le Sport moderne illustré, Paris, Larousse, 1905.

0.. J. LE CAMUS, Les Pratiques aquatiques du bébé, Paris, PUF, 1991.

0.. Thierry TERRET, « Savoir nager. Une histoire des pratiques et des techniques de la natation ", in Techniques sportives et Culture Scolaire sous la direction de P. GOIRAND et J. METZLER, Paris, Éd. Revue EPS, 1991. http://www.ac-amiens.fr/pedagogie/eps/lire/ natation/Descriptionstechniquesnages.htm

0.. P. ARNAUD, Le Militaire, l'écolier, le gymnaste. Naissance de l'éducation physique en France 1869-1889, PUL, 1991, p. 50-67.

0.. Je renvoie ici au texte de J.-F. Bert dans ce même volume.

0.. J.-P. DUMAS, « Aux origines de la méthode naturelle : Georges Hébert et l'enseignement de l'éducation physique à la Marine Française », Revue Internationale d'Histoire Militaire, $\mathrm{n}^{\circ} 83$.

0.. G. ANDRIEU, Georges HÉBERT, dans P. ARNAUD Éd., Le Corps en mouvement. Précurseurs et pionniers en éducation physique, 1981, p. 291-312. Ici, p. 292-293.

0.. G. HÉBERT, Leçon type de natation, Paris, Éd. Vuibert, 1933.

0.. M. MAUSS, « Techniques du corps », Manuel d'Ethnographie, Paris, Payot, 2002 [1947], p. 51 .

0.. Ibid.

0.. C. Pociello, La Science en mouvements. Étienne Marey et Georges Demeny (1870-1920), Paris, PUF, 1999, p. 161-199.

\section{RÉSUMÉS}

Les techniques du corps sont un moyen de sortir de l'opposition stérile entre internalisme et externalisme en posant l'interaction bioculturelle, et pas seulement symbolique, comme un principe de constitution et de réflexivité corporelle.

The Body technics are the mean to leave the bad opposition between internalism and externalism. The cultural interaction define the corporeal subject, not only in symbolic style, but like a principle of body's constitution and reflexivity. 
« Hetero-reflexividad y técnicas del cuerpo. La epistemología fisio-sico-social de Marcel Mauss ». Las técnicas del cuerpo son un medio para salir de la oposición estéril entre internalismo y externalismo planteando como principio de constitución y de reflexividad corporal, tratándose no solo de la interacción simbólica sino también de la interacción biocultural.

Der Begriff der „Körpertechniken“ ist ein Mittel sich von der sterilen Opposition zwischen Internalismus und Externalismus zu befreien. Die biokulturelle Interaktion wird zum Prinzip der körperlichen Beschaffenheit und Reflexivität.

\section{AUTEUR}

\section{BERNARD ANDRIEU}

Bernard Andrieu est professeur en Épistémologie du corps et des pratiques corporelles à la faculté du Sport de l'UHP Nancy 1, Directeur de l'équipe ACCORPS au sein de l'UMR 7117 CNRS Archives Poincaré (Nancy 1-Nancy 2, Metz), Directeur du Master Philosophie \& rationalités Nancy 2, Metz- Luxembourg. 